\title{
A Brief Survey on Nature Inspired Algorithms: Clever Algorithms for Optimization
}

\author{
P.Sindhuja ${ }^{1}$, P.Ramamoorthy ${ }^{2}$ and M. Suresh Kumar $^{3}$ \\ ${ }^{1 \& 3}$ Assistant Professor, ${ }^{2}$ Professor \\ ${ }^{1}$ United Institute of Technology, Coimbatore, Tamil Nadu, India \\ ${ }^{2}$ Sri Ranganadhar Institute of Engineering and Technology, Coimbatore, Tamil Nadu, India \\ E-Mail:sindhujaphd@gmail.com, covairam33@ymail.com, sureshkumar.m1983@gmail.com
}

\begin{abstract}
This paper presents a brief survey on various optimization algorithms. To be more precise, the paper elaborates on clever Algorithms - a class of Nature inspired Algorithms. The Nature Inspired Computing (NIC) is an emerging area of research that focuses on Physics and Biology Based approach to the Algorithms for optimization. The Algorithms briefed in this paper have understood, explained, adapted and replicated the phenomena of Nature to replicate them in the artificial systems. This Cross - fertilisation of Nature Inspired Computing (NIC) and Computational Intelligence (CI) will definitely provide optimal solutions to existing problems and also open up new arenas in Research and Development. This paper briefs the classification of clever algorithms and the key strategies employed for optimization. Keywords: optimization, collective intelligence, nature inspired, bio inspired algorithms
\end{abstract}

\section{INTRODUCTION}

The literal meaning of optimization refers to the art of making something fully effective. This is achieved by finding an alternative with the most cost effective or highest achievable performance under the given constraints. It is by maximizing the desired factors and minimizing the undesired ones optimization is achieved. Real world optimization problems are definitely challenging to solve.

In spite of numerous optimization tools there is no assurance of arriving to an optimal solution. In general, the concept of optimization is a trial and error method. This is a wide area for research where, new algorithms have been developed to check their effectiveness for providing an optimal solution.
A broader classification of Optimization techniques categorises them into Conventional and Unconventional methods. There are enormous numbers of Survey papers on Conventional techniques. Very few surveyed the Unconventional Techniques.

The real beauty of Unconventional or Bio or Nature Inspired algorithms lies in the fact that the sole inspiration of these algorithms is derived from nature. It is a perfect optimizer and a best teacher with enormous and mysterious capabilities inspiring researchers to imitate nature in technology [1].

Algorithm refers to the procedural logic to achieve solutions for the problems in hand. The term Clever Algorithm refers to ambiguously described algorithms or inconsistent algorithms. Clever algorithms are used in fields of computational intelligence and other relevant fields. This paper presents a clear view about the fundamental concepts in Clever Algorithms. A survey of the various classes of Algorithms is presented in this paper and their inherent properties are analysed. The Classification of the Clever Algorithms and their founders are tabulated [3].

\section{STOCHASTIC ALGORITHMS}

This set of algorithms provide various different strategies by which 'better' and varied starting points can be generated and issued to a neighbourhood searching technique for refinement, a process that is repeated with potentially improving or unexplored areas to search.

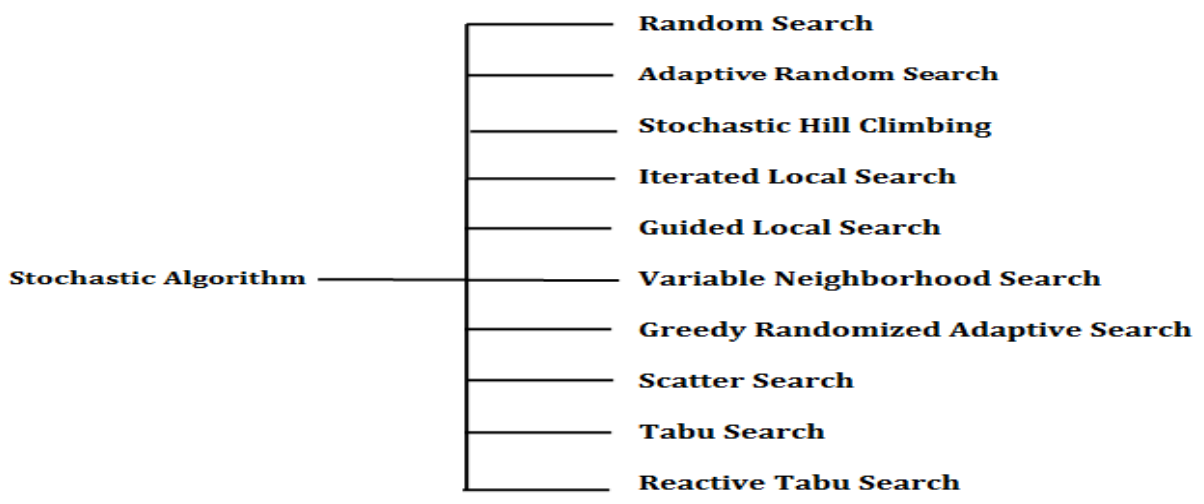

Fig.1 Classification of Stochastic Algorithms 
Random Search does not need any derivatives and is related to techniques that gives minor improvements. It uses uniform probability distribution. The Adaptive Search Algorithm continuously approximates the optimal step size in the search space.

The Stochastic Hill Climbing Algorithm involves iteration of a random selection of neighbour and accepts only if the result is an improvement. The Iterated local Search Algorithm samples a broader neighbourhood and uses local search algorithms to refine the results. The guided local search algorithm uses penalties to use a local search technique and to discover global optima. The search is repeated for a number of times till an optimal solution is attained.

The Variable Neighbourhood Search involves iteration of larger neighbour hoods until an improvement is located. The Greedy Randomized Adaptive Search uses sample stochastically greedy solutions and uses a local search procedure to refine local optima.

The principle behind the Scatter Search approach is that useful information about the global optima is stored in a diverse and elite set of solutions (the reference set).The objective for the Tabu Search algorithm is to constrain an embedded heuristic from returning to recently visited areas of the search space, referred to as cycling. The Reactive Tabu Search addresses this objective by explicitly monitoring the search and reacting to the occurrence of cycles and their repetition by adapting the tabu tenure (tabu list size).
TABLE I AUTHORS OF STOCHASTIC ALgORITHMS

\begin{tabular}{|l|l|}
\hline \multicolumn{2}{|c|}{ STOCHASTIC ALGORITHMS } \\
\hline ALGORITHM & \multicolumn{1}{c|}{ AUTHOR } \\
\hline Random Search & Brooks \\
\hline Adaptive Random Search & Schummer, Steiglitz \\
\hline Stochastic Hill Climbing & Forrest,Mitchell \\
\hline Iterated Local Search & Stuzzle \\
\hline Guided Local Search & Voudouris, Tsang \\
\hline Variable Neighborhood Search & Mladenovic, Hansen \\
\hline Greedy Randomized Adaptive Search & Feo, Resende \\
\hline Scatter Search & Glover \\
\hline Tabu Search & Glover \\
\hline Reactive Search & Battiti, Tecchiolli \\
\hline
\end{tabular}

\section{EVOLUTIONARY ALGORITHMS}

Evolutionary Algorithms deals with the investigation in sophisticated computational systems resembling the simplified versions of mechanisms..

\section{Genetic Algorithm}

Genetic Programming

Evolution Strategies

Differential Evolution

Evolutionary Programming

Evolutionary Algorithms

Grammatical Evolution

Gene Expression Programming

Learner Classifier System

Non - Dominated sorting Genetic Algorithm

Strength Pareto Evolutionary Algorithm

Fig. 2 Classification of Evolutionary Algorithms 
The objective of the Genetic Algorithm is to repeatedly employ surrogates for the recombination and mutation genetic mechanisms on the population of candidate solutions. In Genetic Programming, the Genetic Algorithm employs induction to design a new computer program. Evolution Strategies algorithm maximizes the suitability of collection of candidate solutions in the context of an objective function from a domain. The Differential Evolution algorithm involves maintaining a population of candidate solutions subjected to iterations of recombination, evaluation, and selection. [3]

In Evolutionary Programming algorithm the suitability of a collection of candidate solutions in the context of an objective function from the domain is maximized. The idea behind the Grammatical Evolution is to adapt an executable program to a problem specific objective function. Gene Expression Programming algorithm improves the adaptive fit of an expressed program in the context of a problem specific cost function. The Learning Classifier System algorithm brings out optimization by using a payoff strategy on exposure to stimuli. The Non-dominated Sorting Genetic Algorithm improves the adaptive fit of a population of candidate solutions to a Pareto front constrained by a set of objective functions. Strength Pareto Evolutionary Algorithm locates and maintains a front of non-dominated solutions, ideally a set of Pareto optimal solutions.
TABLE II AUTHORS OF EVOLUTIONARY ALGORITHMS

\begin{tabular}{|l|l|}
\hline \multicolumn{2}{|c|}{ EVOLUTIONARY ALGORITHMS } \\
\hline ALGORITHM & AUTHOR \\
\hline Genetic Algorithm & Holland \\
\hline Genetic Programming: & Cramer \\
\hline Evolution Strategies algorithm & Bienert, Rechenberg, \\
\hline Differential Evolution algorithm & Storn, Price \\
\hline Evolutionary Programming & Lawrence Fogel \\
\hline Grammatical Evolution & Ryan, Collins and \\
\hline Gene Expression Programming & Ferreira \\
\hline Learning Classifier System & Holland \\
\hline Non-dominated Sorting Genetic & Srinivas and Deb \\
\hline Strength Pareto Evolutionary & Zitzler and Thiele \\
\hline
\end{tabular}

\section{PHYSICAL ALGORITHMS}

Physical algorithms are those algorithms inspired by a physical Process

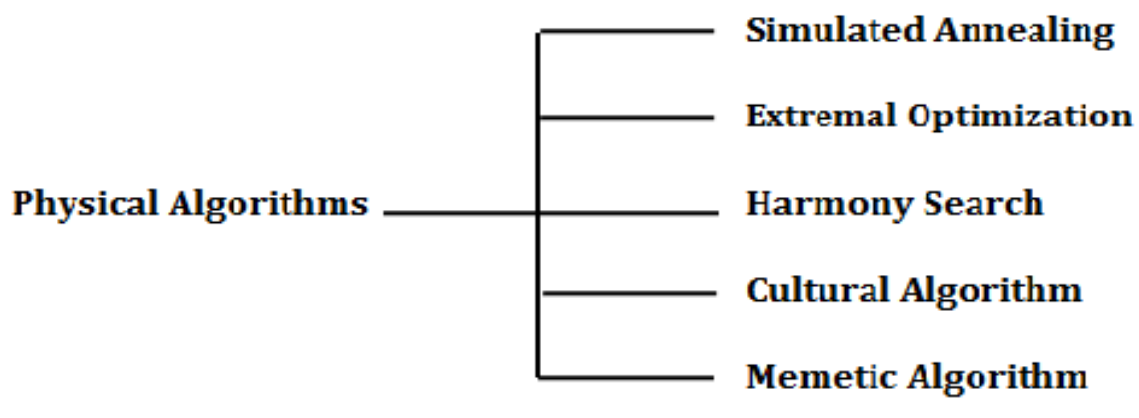

Fig. 3 Classification of Physical Algorithms

In Simulated Annealing the information processing objective of the technique is to locate the minimum cost configuration in the search space. This algorithm re samples the problem space. In Extremal Optimization the dynamics of the system result in the steady improvement of a candidate solution with sudden and large crashes in the quality of the candidate solution. The Harmony Search Algorithm uses the already available good candidate solutions to influence the new candidate solutions. As Evolution happens, individuals share their accumulated information to the other individuals. The cultural Algorithm employs this strategy.[4] The Memetic Algorithm of the information processing strategy is to exploit a population based global search technique to broadly locate good areas of the search space, combined with the repeated usage of a local search heuristic by individual solutions to locate local optimum.
TABLE III AUTHORS OF PHYSICAL ALgORITHMS

\begin{tabular}{|l|l|}
\hline \multicolumn{2}{|c|}{ PHYSICAL ALGORITHMS } \\
\hline \multicolumn{1}{|c|}{ ALGORITHM } & \multicolumn{1}{c|}{ AUTHOR } \\
\hline Simulated Annealing & Kirkpatrick, Gelatt, and Vecchi \\
\hline Extremal Optimization & Boettcher and Percus \\
\hline Harmony Search & Geem et al \\
\hline Cultural Algorithm & Reynolds \\
\hline Memetic Algorithm & Moscato \\
\hline
\end{tabular}




\section{PROBABILISTIC ALGORITHMS}

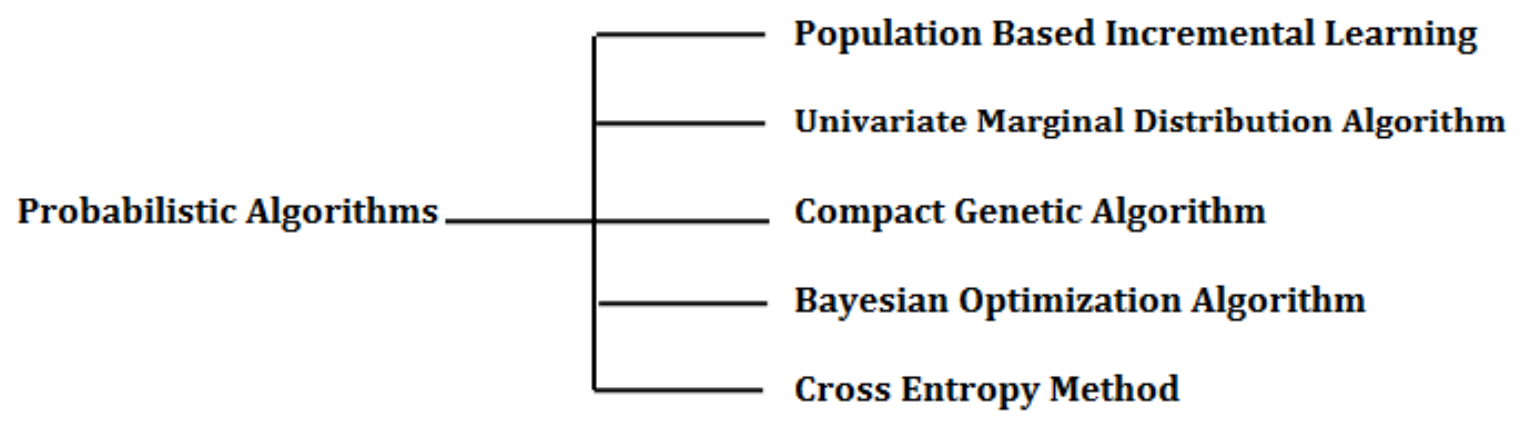

Fig. 4 Classification of Probabilistic Algorithms

Probabilistic Algorithms are those algorithms that model a problem or search a problem space using an probabilistic model of candidate Solutions.

The information processing objective of the PopulationBased Incremental Learning (PBIL) algorithm [3] is to reduce the memory required by the genetic algorithm. This is done by reducing the population of candidate solutions Univariate Marginal Distribution Algorithm. The information processing strategy of the algorithm is to use the frequency of the components in a population of candidate solutions in the construction of new candidate solutions.

The behaviour of a much smaller memory footprint is simulated in a Compact Genetic Algorithm. The Bayesian Optimization Algorithm constructs a probabilistic model that describes the relationships between the components of fit solutions in the problem space. The information processing strategy of the Cross-Entropy Method algorithm is to sample the problem space and approximate the distribution of good solutions.
TABle IV Authors of Probabilistic Algorithms

\begin{tabular}{|c|c|}
\hline \multicolumn{2}{|c|}{ PROBABILISTIC ALGORITHMS } \\
\hline ALGORITHM & AUTHOR \\
\hline Population-Based Incremental & Baluja \\
\hline Univariate Marginal Distribution & M“uhlenbein \\
\hline Compact Genetic Algorithm & Harik, Lobo, and Goldberg \\
\hline Bayesian Optimization Algorithm & Pelikan, Goldberg,and \\
\hline Cross-Entropy Method & De Boer et al \\
\hline
\end{tabular}

\section{SWARM ALGORITHMS}

Swarm intelligence deploys the idea of Collective Intelligence(CI). When a huge number of agents (homogenous) work in cooperation, for the accomplishment of a particular task, it is referred to as Collective Intelligence. Ex - Fish, Ants etc., CI is selforganizing and decentralized. Usually agents use this technique to solve their living issues like search of food, shelter , prey evading etc.,

\section{Population Based Incremental Learning}

Univariate Marginal Distribution Algorithm

Compact Genetic Algorithm

Bayesian Optimization Algorithm

\section{Cross Entropy Method}

Fig. 5 Classification of Swarm Algorithms 
The goal of the Particle Swarm Optimization algorithm achieve particle local optimisation by assigning initially random positions to all particles in the space and small initial random velocities. The Ant System strategy is to exploit historic and heuristic information to construct candidate solutions and fold the information learned from constructing solutions into the history. Ants wander around for food, once it locates the food it lays down pheromone in the environment. Numerous trips are made by additional ants resulting in more pheromone deposition.

A positive feedback process routes more and more ants to productive paths that are in turn further refined through use. This is the idea behind the Ant Colony System. The Bacterial Foraging Algorithm mimics the behaviour of E.coli Bacteria that perceives the direction to food based on the gradients of chemicals in their environment. Similarly, bacteria secrete attracting and repelling chemicals into the environment and can perceive each other in a similar way. Using locomotion mechanisms (such as flagella) bacteria can move around in their environment, sometimes moving chaotically (tumbling and spinning), and other times moving in a directed manner that may be referred to as swimming [3].

\section{Probabilistic Algorithms}

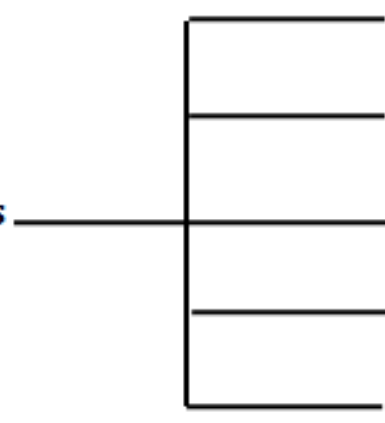

TABLE V AUTHORS OF SWARM ALGORITHMS

\begin{tabular}{|c|c|}
\hline \multicolumn{2}{|c|}{ SWARM ALGORITHMS } \\
\hline ALGORITHM & AUTHOR \\
\hline Particle Swarm Optimization & Eberhart and Kennedy \\
\hline Ant System & Dorigo, Maniezzo, and Colorni \\
\hline Ant Colony System & Dorigo and Gambardella \\
\hline Bees Algorithm & Pham et al \\
\hline Bacterial Foraging Algorithm & Liu and Passino \\
\hline
\end{tabular}

\section{IMMUNE ALGORITHMS}

A simplified description of the immune system is an organ system intended to protect the host organism from the threats posed to it from pathogens and toxic substances. Pathogens encompass a range of microorganisms such as bacteria, viruses, parasites and pollen.

\section{Population Based Incremental Learning}

Univariate Marginal Distribution Algorithm

\section{Compact Genetic Algorithm}

Bayesian Optimization Algorithm

\section{Cross Entropy Method}

Fig. 6 Classification of Immune Algorithms

The information processing principles of the clonal selection theory describe a general learning strategy. This strategy involves a population of adaptive information units (each representing a problem-solution or component) subjected to a competitive processes for selection, which together with the resultant duplication and variation ultimately improves the adaptive fit of the information units to their environment [3].

The Negative Selection Algorithm uses self non self discrimination process via negative selection. The Artificial Immune Recognition system begins with an initial collection of general immune cells in response to the environment.

A fascinating network theory and signalling information is available with immune network theory. The Dendritic Cell Algorithm prepares a set of dendritic cells that provides context specific information about how to classify input patterns.
TABLE VI AUTHORS OF IMMUNE ALGORITHMS

\begin{tabular}{|c|c|}
\hline \multicolumn{2}{|c|}{ IMMUNE ALGORITHMS } \\
\hline ALGORITHM & AUTHOR \\
\hline Clonal Selection Algorithm & de Castro and Von Zuben \\
\hline Negative Selection Algorithm & Forrest et al. \\
\hline Artificial Immune Recognition & Watkins \\
\hline Immune Network Algorithm & Farmer et al. \\
\hline Dendritic Cell Algorithm & Greensmith, Aickelin and \\
\hline
\end{tabular}

\section{NEURAL ALGORITHMS}

A Biological Neural Network refers to the elements in the nervous system, consisting of neurons communicating with each other through electrochemical signals. This idea of communication gave rise to the following list of Neural Algorithms. 
TABle VII Authors of NeURAl Algorithms

\begin{tabular}{|c|c|}
\hline \multicolumn{2}{|c|}{ NEURAL ALGORITHMS } \\
\hline ALGORITHM & AUTHOR \\
\hline Perceptron & Rosenblatt \\
\hline Back Propagation & Bryson and Hoin \\
\hline Hopfield Network & Hopfield \\
\hline Learning Vector Quantization & Kohonen, Barna, and Chrisley \\
\hline Self-Organizing Map & Kohonen \\
\hline
\end{tabular}

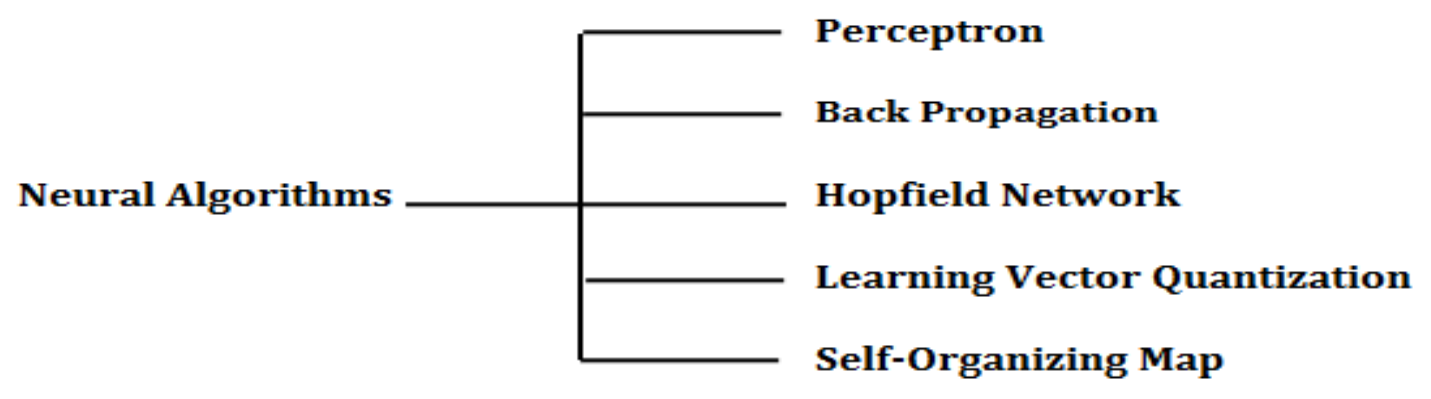

Fig. 7 Classification of Neural Algorithms

The information processing objective of the Perceptron technique is to model a given function by modifying internal weightings of input signals to produce an expected output signal. The system is trained using a supervised learning method, where the error between the system's output and a known expected output is presented to the system and used to modify its internal state. The Back Propagation algorithm, one of the oldest techniques of Neural Networks models a given function by modifying the internal weightings of the input signal to produce the expected output signal. The Hopfield pattern associates the components of an input pattern with a holistic representation of the pattern called Content Addressable Memory (CAM). The Learning Vector Quantization prepares a set of codebook (or prototype) vectors in the domain of the observed input data samples and to use these vectors to classify unseen examples.[5] The Self-Organizing Map optimally places a topology (grid or lattice) of codebook or prototype vectors in the domain of the observed input data samples.

\section{CONCLUSION}

It is obvious that the field of Clever Algorithms is rapidly growing. The Unconventional methods of Optimization are rapidly exploited areas of research. This paper presents a critical survey of clever algorithms. Clever algorithms are used extensively not only in artificial intelligence but also in automation in other real life domains like military, healthcare, manufacturing, mining etc. There are many practical concerns with this clever algorithms like ensuring the correct implementation of the algorithms, issues regarding which software platform to use for implementing such algorithms, how to test the validity of the algorithms, issues to consider while comparing the clever algorithms. These aspects are actively being looked upon.

\section{REFERENCES}

[1] Ji`rí Kubalík, "Biologically Inspired Algorithms (A4M33BIA) Optimization: Conventional and Unconventional Optimization Techniques: Introduction to Soft Computing optimization”, 2008

[2] Raymond Chiong, "Nature Inspired Algorithms for optimization", Springer Journal of Computation Intelligence and Complexit, Ed., 2009.

[3] Shilpi Gupta, Shweta Bhardwaj and Parul Kalra Bhatia, ““”A reminiscent study of nature inspired computation”, International Journal of Advances in Engineering \&amp; Technology, May 2011.

[4] Jason Brownlee PhD, "Clever Algorithms Nature-Inspired Programming Recipes”, 2011

[5] S.Binitha and S. Siva Sathya, "A Survey of Bio inspired Optimization Algorithms", International Journal of Soft Computing and Engineering (IJSCE) ISSN: 2231-2307, Vol. 2, No. 2, May 2012

[6] Iztok Fister Jr., Xin-She Yang, Iztok Fister, Janez Brest, Dušan Fister arXiv:1307.4186 [cs.NE] , "A Brief Review of Nature-Inspired Algorithms for Optimization”, July 2013

[7] N.Siddique and H. Adeli, "Nature Inspired Computing: An Overview and Some Future Directions", Springer Journal of Cogn Comput, Vol.7, pp.706,2015.

[8] Manas Kumar Yogi1 Darapu Uma, “A Survey of Nature Inspired Clever Algorithms”, International Journal for Scientific Research and Development, Vol. 4, No. 07, 2016.

[9] Surjit Singh and Rajeev Mohan Sharma, "Optimization Techniques in Wireless Sensor Networks”, ACM Digital Library, March 4-5,2016. 